\title{
CORRIGENDUM
}

\section{TSPY and its X-encoded homologue interact with cyclin B but exert contrasting functions on cyclin-dependent kinase 1 activities}

\author{
Y Li and Y-F Chris Lau \\ Oncogene (2009) 28, 1879; doi:10.1038/onc.2009.64
}

Correction to: Oncogene (2008) 27, 6141-6150; doi:10.1038/onc.2008.206; published online 30 June 2008

Since the publication of the above manuscript the authors have identified an error in Figure 6. The +

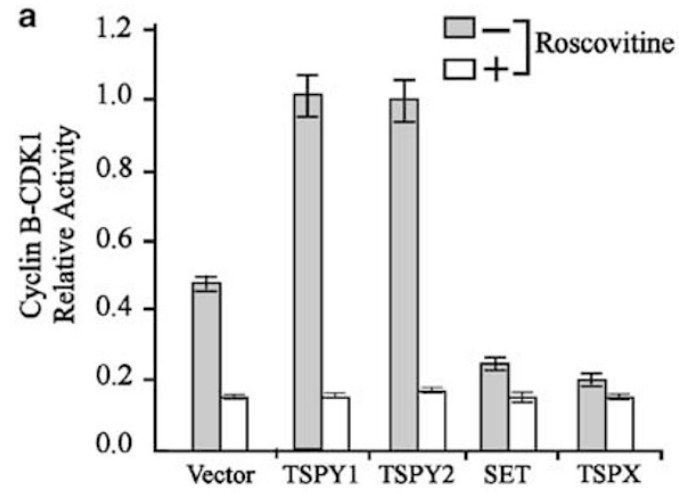

b

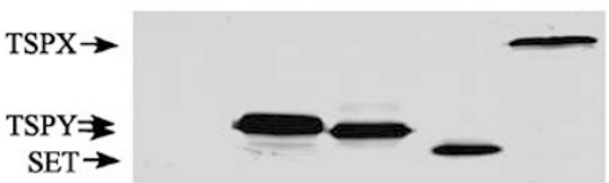

C

Cyclin B1

CDK1

Tubulin

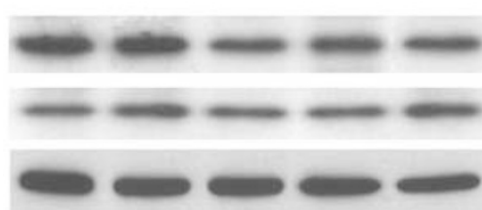

Figure 6 In vivo effects of testis-specific protein Y-encoded (TSPY), SET and TSPX on cyclin B-CDK1 activities in transfected cells. (a) The in vivo cyclin B1-CDK1 activities were analysed by the CycLex Cdc2-Cyclin B kinase assays using total cell lysates from respective HEK293 transfected cells. Expression of FLAG-tagged TSPY type 1 (TSPY1) or type 2 (TSPY2) protein in HEK293 cells enhanced the endogenous cyclin B1-CDK1 activities, whereas similar expression of SET or TSPX protein repressed the same kinase complex. In parallel assays, the presence of roscovitine, an inhibitor of cyclin B1-CDK1 kinase, abolished the cyclin B-CDK1 activities to the same level among the same cell lysates. (b) Western blotting of total cell lysates showing the expression of TSPY, SET and TSPX in the respectively transfected cell populations. (c) Western blotting of the corresponding total cell lysates demonstrating the presence of endogenous cyclin B1 (top row) and CDK1 (middle row) proteins and tubulin control (bottom row). and - signs, indicating the presence and absence of Roscovitine, were mistakenly reversed. The corrected version of the figure is given here. 